\title{
Microwave Imaging of Three-Dimensional Targets by Means of an Inexact-Newton-Based Inversion Algorithm
}

\author{
Claudio Estatico, ${ }^{1}$ Matteo Pastorino, ${ }^{2}$ and Andrea Randazzo ${ }^{2}$ \\ ${ }^{1}$ Department of Mathematics, University of Genoa, Via Dodecaneso 35, 16146 Genova, Italy \\ ${ }^{2}$ Department of Naval, Electrical, Electronic, and Telecommunication Engineering, University of Genoa, Via Opera Pia 11A, \\ 16145 Genova, Italy
}

Correspondence should be addressed to Andrea Randazzo; andrea.randazzo@unige.it

Received 10 May 2013; Accepted 16 July 2013

Academic Editor: Qing Huo Liu

Copyright (C) 2013 Claudio Estatico et al. This is an open access article distributed under the Creative Commons Attribution License, which permits unrestricted use, distribution, and reproduction in any medium, provided the original work is properly cited.

A microwave imaging method previously developed for tomographic inspection of dielectric targets is extended to threedimensional objects. The approach is based on the full vector equations of the electromagnetic inverse scattering problem. The ill-posedness of the problem is faced by the application of an inexact-Newton method. Preliminary reconstruction results are reported.

\section{Introduction}

Microwave imaging is a technique aimed at inspecting unknown targets by using interrogating electromagnetic waves at microwave frequencies. As it is well known, the image formation is based on the solution of an electromagnetic inverse scattering problem. One of the main features of microwave imaging is the ability of providing the distributions of the dielectric parameters (e.g., dielectric permittivity and electric conductivity), which cannot be directly retrieved by using other diagnostic techniques [1-18]. From a theoretical point of view, this achievement can be obtained even in the case of three-dimensional (3D) configurations. As a matter of fact, some initial works in this area were just oriented to 3D imaging [19-26]. Unfortunately, the computational resources needed to face a full vector $3 \mathrm{D}$ inverse scattering problem has been prohibitive for a long time. Consequently, the research community mainly focused his efforts on two-dimensional (2D) settings. In particular, the presence of cylindrical targets (uniform and homogeneous along an infinite direction) has been usually assumed, leading to the well known microwave tomography [27-49].

More recently, the enormous improvements in computer powers have allowed reconsidering the $3 \mathrm{D}$ formulation. In addition, new efficient solving methods have been developed in the past years. Most of them allow a more or less straightforward extension for the inspection of 3D targets. Some examples of these approaches are reported in [50-69] and in the references therein.

In this context, the present paper introduces for the first time an extension to 3D scatterers of a diagnostic tomographic approach recently developed by the authors [70-74]. It is based on a two-step inexact-Newton method for the solution of two electric field integral equations (EFIEs), namely the data equation and the state equation, which represent the physical model of the whole scattering process. By a theoretical point of view, the approach is quite a classic one. Anyway, the particular inexact-Newton iterative solving procedure has been found to be quite robust against the illposedness of the considered inverse scattering problem both by assuming synthetic or experimental data.

In the 3D case under investigation, the full vector equations, written in terms of the Green's dyadic function for free space, are discretized and combined in order to obtain a single functional equation, which is iteratively solved by Gauss-Newton linearization (outer step) and successive regularization by truncated Landweber method (inner step).

The paper is organized as follows. The mathematical formulation of the developed approach is discussed in Section 2. Section 3 reports some initial numerical results aimed at validating the inversion procedure. Finally, conclusions are drawn in Section 4. 


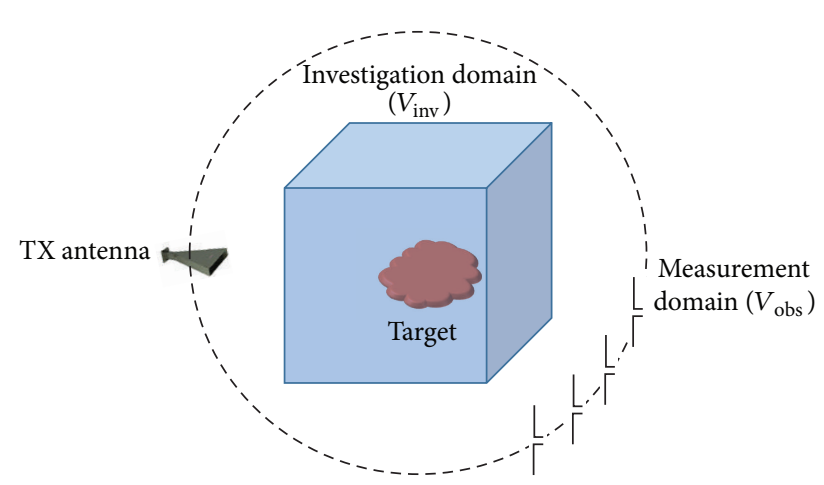

FIGURE 1: Imaging configuration.

\section{Mathematical Formulation}

Let us consider the configuration of Figure 1. An unknown dielectric target is located inside a predefined volume (in the following denoted as investigation domain, $V_{\text {inv }}$ ). The object is illuminated by one or more incident electric fields $\mathbf{E}_{\text {inc }}(\mathbf{r})$ (generated by proper antennas located outside the investigation area). The total electric field $\mathbf{E}_{\text {tot }}(\mathbf{r})$ produced by the interaction between the target and the illuminating radiation is collected by means of RX antennas located in a measurement domain $V_{\text {obs }}$. As we will better analyze in Section 3, we mention that in our numerical simulation the investigation domain is a cube, while the TX and RX antennas are uniformly distributed on an outside sphere of predefined radius.

For sake of simplicity, a single-view case is described in the following. The extension of the mathematical formulation to the multiview case is however straightforward. Moreover, a $e^{j \omega t}$ time dependence is assumed and the corresponding terms are omitted in the following.

2.1. Electromagnetic Model. The scattered electric field $\mathbf{E}_{\text {scatt }}(\mathbf{r})=\mathbf{E}_{\text {tot }}(\mathbf{r})-\mathbf{E}_{\text {inc }}(\mathbf{r})$ is related to the dielectric properties of the investigated area $V_{\text {inv }}$ by means of the following two integral equations [1], usually referred to as data and state equations,

$$
\begin{gathered}
\mathbf{E}_{\text {scatt }}(\mathbf{r})=\mathbf{G}_{\text {data }}\left(c \mathbf{E}_{\text {tot }}\right)(\mathbf{r}), \quad \mathbf{r} \in V_{\text {obs }}, \\
\mathbf{E}_{\text {inc }}(\mathbf{r})=\mathbf{E}_{\text {tot }}(\mathbf{r})-\mathbf{G}_{\text {state }}\left(c \mathbf{E}_{\text {tot }}\right)(\mathbf{r}), \quad \mathbf{r} \in V_{\text {inv }},
\end{gathered}
$$

where $c(\mathbf{r})=\epsilon_{r}(\mathbf{r})-1$ is the contrast function (with $\epsilon_{r}$ being the space dependent relative complex dielectric permittivity of the investigation domain) and the linear operators $\mathbf{G}_{\text {data }}$ and $\mathbf{G}_{\text {state }}$ are defined as

$$
\begin{array}{ll}
\mathbf{G}_{\text {data }} \mathbf{f}(\mathbf{r})=-k_{0}^{2} \int_{V_{\mathrm{inv}}} \mathbf{f}(\mathbf{r}) \cdot \overline{\mathbf{G}}_{0}\left(\mathbf{r}, \mathbf{r}^{\prime}\right) d \mathbf{r}^{\prime}, & \mathbf{r} \in V_{\mathrm{obs}}, \\
\mathbf{G}_{\text {state }} \mathbf{f}(\mathbf{r})=-k_{0}^{2} \int_{V_{\mathrm{inv}}} \mathbf{f}(\mathbf{r}) \cdot \overline{\mathbf{G}}_{0}\left(\mathbf{r}, \mathbf{r}^{\prime}\right) d \mathbf{r}^{\prime}, & \mathbf{r} \in V_{\mathrm{inv}}
\end{array}
$$

with $k_{0}=\omega \sqrt{\epsilon_{0} \mu_{0}}$ being the free-space wavenumber and

$$
\overline{\mathbf{G}}_{0}\left(\mathbf{r}, \mathbf{r}^{\prime}\right)=-\frac{1}{4 \pi}\left(\overline{\mathbf{I}}+\frac{\nabla \nabla}{k_{0}^{2}}\right) \frac{e^{-j k\left|\mathbf{r}-\mathbf{r}^{\prime}\right|}}{\left|\mathbf{r}-\mathbf{r}^{\prime}\right|}
$$

the free-space dyadic Green's function.
The data and state equations can be merged in order to obtain a single nonlinear operator equation relating the contrast function and the scattered electric field as follows

$$
\mathbf{E}_{\text {scatt }}(\mathbf{r})=\mathbf{G}_{\text {data }}\left(\mathbf{C}\left(\left(\mathbf{I}-\mathbf{G}_{\text {state }} \mathbf{C}\right)^{-1} \mathbf{E}_{\text {inc }}\right)\right)(\mathbf{r}),
$$

where $\mathbf{r} \in V_{\mathrm{obs}}$ and $\mathbf{C}$ is the operator such that $\mathbf{C}(\mathbf{f})(\mathbf{r})=$ $c(\mathbf{r}) \mathbf{f}(\mathbf{r}), \mathbf{r} \in V_{\text {inv }}$. By defining the nonlinear operator $\mathbf{F}(c)=$ $\mathbf{G}_{\text {data }}\left(\mathbf{C}\left(\left(\mathbf{I}-\mathbf{G}_{\text {state }} \mathbf{C}\right)^{-1} \mathbf{E}_{\text {inc }}\right)\right)$ that maps the contrast function $c$ with the scattered electric field $\mathbf{E}_{\text {scatt }}$, (4) can be written in compact form as follows:

$$
\mathbf{F}(c)(\mathbf{r})=\mathbf{E}_{\text {scatt }}(\mathbf{r}), \quad \mathbf{r} \in V_{\text {obs }} .
$$

The nonlinear equation (5) models the full inverse scattering problem: given the scattered electric field $\mathbf{E}_{\text {scatt }}$ (i.e., the data), measured in the measurement domain $V_{\text {obs }}$, find the contrast function $c$ (i.e., the unknown) in the investigation domain $V_{\text {inv }}$ such that $\mathbf{F}(c)=\mathbf{E}_{\text {scatt }}$.

2.2. Regularized Inversion Algorithm. The integral equation (5) for the computation of the contrast function $c$ turns out to be ill posed, and its solution requires a regularization algorithm. To this end, the inner/outer regularizing scheme developed in [70-72] for the 2D case is here generalized to a 3D configuration. The inner/outer scheme can be summarized as follows. Any outer step is a basic GaussNewton linearization of the nonlinear equation (5). Such a linearized equation is then solved by means of the truncated Landweber iterative method, which represents the inner step.

In particular, the algorithm works as follows.

(1) Initialize the outer loop by setting $k=0$ ( $k$ denotes the outer iteration index) and by choosing a starting guess $c_{0}$ (if no a priori information is available, just void domain can be used, i.e., $c_{0}=0$ ).

(2) Linearize (5) in order to obtain the following linear equation ("outer loop")

$$
\mathbf{F}_{c_{k}}^{\prime} h_{k}(\mathbf{r})=\mathbf{E}_{k}(\mathbf{r}), \quad \mathbf{r} \in V_{\mathrm{obs}},
$$

where $\mathbf{F}_{c_{k}}^{\prime}$ is the Frechét derivative of $\mathbf{F}$ at $c_{k}$ and $\mathbf{E}_{k}(\mathbf{r})=\mathbf{E}_{\text {scatt }}(\mathbf{r})-\mathbf{F}\left(c_{k}\right)(\mathbf{r}), \mathbf{r} \in V_{\text {obs }}$.

(3) Compute a regularized solution of the linear equation (6) with respect to the unknown $h_{k}(\mathbf{r}), \mathbf{r} \in V_{\text {inv }}$, by using the following truncated Landweber algorithm ("inner loop", where $l$ denotes the corresponding inner iteration index)

$$
\begin{gathered}
h_{k, 0}(\mathbf{r})=0, \\
h_{k, l+1}(\mathbf{r})=h_{k, l}(\mathbf{r})-\beta \mathbf{F}_{c_{k}}^{\prime^{*}}\left(\mathbf{F}_{c_{k}}^{\prime} h_{k, l}(\mathbf{r})-\mathbf{E}_{k}(\mathbf{r})\right),
\end{gathered}
$$

where $\beta=\left\|\mathbf{F}_{c_{k}}^{\prime *} \mathbf{F}_{c_{k}}^{\prime}\right\|^{-1}$ is a constant values. Here $\mathbf{F}_{c_{k}}^{\prime^{*}}$ denotes the adjoint operator of $\mathbf{F}_{c_{k}}^{\prime}$. The inner iterations are stopped when a maximum number of iterations $L$ is reached. 
(4) Update the current solution as

$$
c_{k+1}(\mathbf{r})=c_{k}(\mathbf{r})+h_{k, L}(\mathbf{r}) .
$$

(5) Set $k=k+1$ and iterate from step (2) until a predefined stopping rule (such as discrepancy principle or GCV) is satisfied or a maximum number of outer iterations $K$ is reached.

We briefly recall that the number of inner step $L$ acts as the regularization parameter of the truncated Landweber algorithm for the solution of any Newton equation (6). Basically, during the first iterations the Landweber method is able to filter out the components usually corrupted by noise (i.e., the highest frequencies Fourier components of the data $\mathbf{E}_{k}$ ), so that an early stop of the inner iterations gives rise to a regularization effect [71].

Similarly to the two-dimensional case, the Frechét derivative of the operator $\mathbf{F}$ is given by

$$
\mathbf{F}_{c}^{\prime} h(\mathbf{r})=\mathbf{G}_{\text {data }}^{c}\left(h \mathbf{E}_{\text {tot }}^{c}\right)(\mathbf{r}), \quad \mathbf{r} \in V_{\text {obs }},
$$

where $\mathbf{E}_{\text {tot }}^{c}(\mathbf{r})=\left(\left(\mathbf{I}-\mathbf{G}_{\text {state }} \mathbf{C}\right)^{-1} \mathbf{E}_{\text {inc }}\right)(\mathbf{r}), \mathbf{r} \in V_{\text {inv }}$, and the operator $\mathbf{G}_{\text {data }}^{c}$ is given by

$$
\mathbf{G}_{\text {data }}^{c} \mathbf{f}(\mathbf{r})=-k_{0}^{2} \int_{V_{\text {inv }}} \mathbf{f}(\mathbf{r}) \cdot \overline{\mathbf{G}}_{c}\left(\mathbf{r}, \mathbf{r}^{\prime}\right) d \mathbf{r}^{\prime}, \quad \mathbf{r} \in V_{\mathrm{obs}}
$$

with $\overline{\mathbf{G}}_{c}$ being the dyadic Green's function for an inhomogeneous background characterized by a contrast function $c$.

2.3. Discretization of the Scattering Equations. The data and state scattering equations (1) are discretized by using pulse basis functions and Dirac's delta weighting functions [75]. In particular, the investigation domain $V_{\text {inv }}$ is discretized into $N$ cubic voxels of side $l$ and centers $\mathbf{r}_{n}^{\text {inv }}, n=1, \ldots, N$, and the observation domain is composed by $M$ measurement points located at positions $\mathbf{r}_{m}^{\text {meas }}, m=1, \ldots, M$.

Consequently, the discrete version of (4) is

$$
\mathbf{e}_{\text {scatt }}=\mathbf{G}_{\text {data }} D_{3}(\mathbf{c})\left(\mathbf{I}-\mathbf{G}_{\text {state }} D_{3}(\mathbf{c})\right)^{-1} \mathbf{e}_{\text {inc }},
$$

where $\mathbf{c}=\left[c\left(\mathbf{r}_{1}^{\text {inv }}\right) \cdots c\left(\mathbf{r}_{N}^{\text {inv }}\right)\right]^{t}$ is an array containing the values of the contrast function inside the voxels,

$$
\mathbf{e}_{\text {tot } / \text { scatt } / \text { inc }}=\left[\begin{array}{l}
\mathbf{e}_{\text {tot } / \text { scatt } / \text { inc }}^{x} \\
\mathbf{e}_{\text {tot } / \text { scatt } / \text { inc }} \\
\mathbf{e}_{\text {tot } / \text { scatt } / \text { inc }}^{z}
\end{array}\right]
$$

with $\quad \mathbf{e}_{\text {tot } / \text { inc }}^{x / y / z}=\left[E_{\text {tot } / \text { inc }}^{x / y / z}\left(\mathbf{r}_{1}^{\text {inv }}\right) \cdots E_{\text {tot } / \text { inc }}^{x / y / z}\left(\mathbf{r}_{N}^{\text {inv }}\right)\right]^{t}$ and $\mathbf{e}_{\text {scatt }}^{x / y / z}=$ $\left[E_{\text {scatt }}^{x / y / z}\left(\mathbf{r}_{1}^{\text {meas }}\right) \cdots E_{\text {scatt }}^{x / y / z}\left(\mathbf{r}_{M}^{\text {meas }}\right)\right]^{t}$ (the superscript denotes the components of the field vector), and $D_{3}(\cdot)$ is the discrete diagonal operator defined as

$$
D_{3}(\mathbf{c})=\left[\begin{array}{lllllllll}
c_{1} & & & & & & & & \\
& \ddots & & & & & & 0 & \\
& & c_{N} & & & & & & \\
& & & c_{1} & & & & & \\
& & & & \ddots & & & & \\
& & & & & c_{N} & & & \\
& & & & & & c_{1} & & \\
& & & & & & & & \ddots \\
& & & & & & & c_{N}
\end{array}\right] .
$$

In (11) the matrices $\mathbf{G}_{\text {data }}$ and $\mathbf{G}_{\text {state }}$ are the discrete counterparts of the linear operators defined in (2) and they contain the integrals of the components of the corresponding dyadic Green's function over any voxels' volume [76]. The discrete version of the Frechét derivative used in (6) is obtained in a similar way.

It is worth noting that, in the numerical implementation of the approach, a BiCGStab-FFT approach $[77,78]$ has been used to speed up the computation of the total internal field and of the inhomogeneous dyadic Green's function.

\section{Numerical Results}

The developed approach has been tested by means of several numerical simulations. In all cases, a plane wave illumination is assumed. Moreover, a multiview configuration, in which the object is illuminated by subsequent different waves impinging from $N_{S}$ uniformly distributed directions, is used in order to increase the available information. The scattered field is collected into $N_{M}$ measurement points uniformly distributed on a sphere of radius $R_{M}$ for any impinging wave. The synthetic data used for the inversion have been obtained by using a numerical simulator based on the Method of Moments [75]. A finer discretization than that used in the inversion code is employed in order to avoid inverse crimes. Moreover, the computed data have been corrupted with a Gaussian noise with zero-mean value and variance corresponding to a signal-to-noise ratio SNR.

As a first case, a single homogeneous cubic target is considered. The center of the object is located in $\mathbf{r}_{c}=$ $\left(0.1 \lambda_{0}, 0.1 \lambda_{0}, 0.1 \lambda_{0}\right)$, whereas its radius and relative dielectric permittivity are equal to $l=0.5 \lambda_{0}$ and $\epsilon_{r}=2.0$, respectively. The investigation area is a cubic volume of side $D=\lambda_{0}$, which has been partitioned into $N=20 \times 20 \times 20=8000$ subdomains. The number of views is $N_{S}=6$, whereas the number of measurement points is $N_{M}=82$ and the radius of the measurement sphere is $R_{M}=\lambda_{0}$. The maximum number of allowed outer iterations has been set equal to $K=20$. The performance of the approach have been firstly analyzed with respect to the signal-to-noise ratio and to the number of iterations of the inner loop. The following two error measures are used:

$$
\begin{gathered}
e_{\text {data }}=\sqrt{\frac{\sum_{s=1}^{S}\left\|\mathbf{e}_{\text {scatt }}-F(\mathbf{c})\right\|^{2}}{\sum_{s=1}^{S}\left\|\mathbf{e}_{\text {scatt }}\right\|^{2}}}, \\
e_{\text {RMSE }}=\frac{\left\|\mathbf{c}-\mathbf{c}_{\text {actual }}\right\|}{\left\|\mathbf{c}_{\text {actual }}\right\|} .
\end{gathered}
$$

The first error measure, $e_{\text {data }}$, can be used in real setting for implementing the discrepancy principle or the GCV, while the second one, $e_{\mathrm{RMSE}}$, can be used only for numerical testing, since it requires the knowledge of the target $\mathbf{c}_{\text {actual }}$.

Table 1 reports the errors obtained for the considered cases and the number of outer iterations needed to reach the best reconstruction. As expected, with noiseless data the inversion algorithm is always able to converge to a correct solution and a higher value of $L$ allows to obtain slightly better 
TABLE 1: Error measures for different values of the signal-to-noise ratio and of the number of inner iterations. Homogeneous dielectric cube.

\begin{tabular}{|c|c|c|c|c|}
\hline SNR & $L$ & $e_{\text {data }}$ & $e_{\mathrm{RMSE}}$ & $K_{\mathrm{opt}}$ \\
\hline \multirow{6}{*}{$\infty$} & 1 & 0.088 & 0.57 & 20 \\
\hline & 2 & 0.06 & 0.54 & 20 \\
\hline & 3 & 0.046 & 0.52 & 20 \\
\hline & 4 & 0.038 & 0.52 & 20 \\
\hline & 5 & 0.032 & 0.51 & 20 \\
\hline & 10 & 0.027 & 0.51 & 20 \\
\hline \multirow{6}{*}{30} & 1 & 0.48 & 0.64 & 20 \\
\hline & 2 & 0.49 & 0.65 & 12 \\
\hline & 3 & 0.48 & 0.65 & 9 \\
\hline & 4 & 0.48 & 0.66 & 8 \\
\hline & 5 & 0.48 & 0.67 & 8 \\
\hline & 10 & 0.48 & 0.72 & 6 \\
\hline \multirow{6}{*}{20} & 1 & 0.62 & 0.66 & 16 \\
\hline & 2 & 0.62 & 0.67 & 11 \\
\hline & 3 & 0.61 & 0.68 & 8 \\
\hline & 4 & 0.62 & 0.68 & 6 \\
\hline & 5 & 0.62 & 0.69 & 6 \\
\hline & 10 & 0.61 & 0.74 & 6 \\
\hline \multirow{6}{*}{10} & 1 & 0.87 & 0.73 & 7 \\
\hline & 2 & 0.89 & 0.75 & 5 \\
\hline & 3 & 0.88 & 0.76 & 4 \\
\hline & 4 & 0.89 & 0.88 & 2 \\
\hline & 5 & 0.88 & 0.81 & 3 \\
\hline & 10 & 0.91 & 0.91 & 2 \\
\hline
\end{tabular}

TABLE 2: Error measures for different values of the number of views. Homogeneous dielectric cube.

\begin{tabular}{lccc}
\hline$N_{S}$ & $e_{\text {data }}$ & $e_{\text {RMSE }}$ & $K_{\text {opt }}$ \\
\hline 6 & 0.52 & 0.66 & 5 \\
12 & 0.54 & 0.57 & 9 \\
18 & 0.56 & 0.55 & 9 \\
29 & 0.54 & 0.53 & 12 \\
\hline
\end{tabular}

TABLE 3: Error measures for different values of the number of measurement points. Homogeneous dielectric cube.

\begin{tabular}{lccc}
\hline$N_{M}$ & $e_{\text {data }}$ & $e_{\text {RMSE }}$ & $K_{\text {opt }}$ \\
\hline 82 & 0.53 & 0.67 & 6 \\
122 & 0.48 & 0.64 & 8 \\
170 & 0.46 & 0.61 & 12 \\
212 & 0.43 & 0.58 & 13 \\
\hline
\end{tabular}

results. On the contrary, when noise is present, low values of $L$ provide a stronger regularization, thus allowing for a better reconstruction. It is worth noting that in these cases, a higher number of iterations are needed to reach the best solution, leading to a higher computational time.
TABLE 4: Error measures for different values of the relative dielectric permittivity. Homogeneous dielectric cube.

\begin{tabular}{lccc}
\hline$\varepsilon_{r}$ & $e_{\text {data }}$ & $e_{\text {RMSE }}$ & $K_{\text {opt }}$ \\
\hline 1.5 & 0.78 & 0.7 & 3 \\
1.75 & 0.63 & 0.66 & 6 \\
2 & 0.53 & 0.64 & 10 \\
2.25 & 0.44 & 0.61 & 14 \\
2.5 & 0.38 & 0.59 & 16 \\
\hline
\end{tabular}

Moreover, Figure 2 shows the behavior of the error measures versus the number of outer iterations and for different values of $L$. The semiconvergence [79] effect is clearly visible in Figure 2(b), confirming that lower values of $L$ provide a stronger regularization, leading however to a higher number of iterations needed to reach convergence.

An example of the reconstructed distribution of the relative dielectric permittivity, concerning the case in which $\mathrm{SNR}=20 \mathrm{~dB}$ and $L=3$, is reported in Figure 3. As can be seen, the target is correctly identified. As expected, the shape is smoothed due to the regularization effect of the inversion algorithm.

The effects of the number of views and of the measurement point number on the quality of the reconstruction have also been evaluated. The obtained results are summarized in Table 2 (errors versus number of views) and Table 3 (errors versus number of measurement points). As expected, as the number of views or the number of measurement points increases, the reconstruction error decreases. However, the computational time increases as well (since the dimensions of the matrices are higher and more iterations are needed).

Finally, the performance of the inversion approach with respect to the value of the relative dielectric permittivity of the target has been evaluated, too. The obtained results are reported in Table 4 . As can be seen, in all the considered cases the developed algorithm provides comparable reconstruction results. However, as expected, for the higher values of dielectric permittivity, a higher number of iterations is required for obtaining a good solution. This is basically due to the effects of the Newton linearization, which is less accurate for strong scatterers (i.e., large values dielectric permittivity), with respect to the original nonlinear operator.

In the second case, a nonhomogeneous structure has been considered. The target is a cube with a void inclusion. The parameters of the cube are as follows: $l=0.9 \lambda_{0}$, center $\mathbf{r}_{c}=$ $(0,0,0)$, and $\epsilon_{r}=1.5$. The inclusion has a spherical shape with radius $r_{s}=0.25 \lambda_{0}$ and center $\mathbf{r}_{s}=(0,0,0)$. The investigation area is a cubic volume of side $D=1.5 \lambda_{0}$, which has been partitioned into $N=28 \times 28 \times 28=21952$ subdomains. The maximum number of allowed outer iterations has been set equal to $K=20$, whereas the number of inner iterations is $L=2$. The number of views is $N_{S}=6$, whereas $N_{M}=$ 101 measurement points uniformly distributed on a sphere of radius $R_{M}=1.5 \lambda_{0}$ has been considered. The distribution of the reconstructed dielectric permittivity is shown in Figure 4. As can be seen, the reconstruction algorithm provides a good 


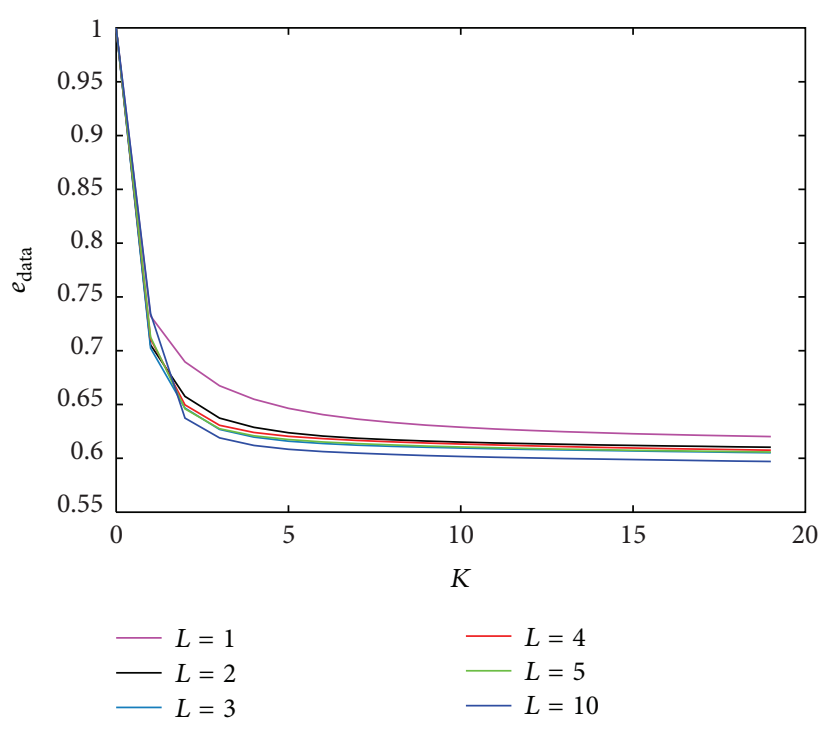

(a)

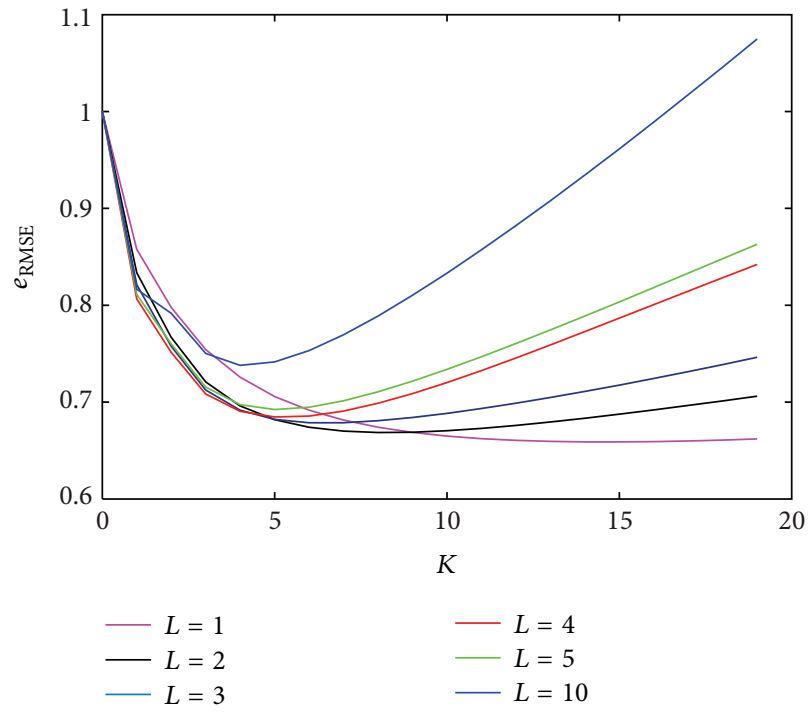

(b)

FIGURE 2: Error measures versus the number of outer iterations for different values of the number of inner iterations. Homogeneous dielectric cube. $\mathrm{SNR}=20 \mathrm{~dB}$.

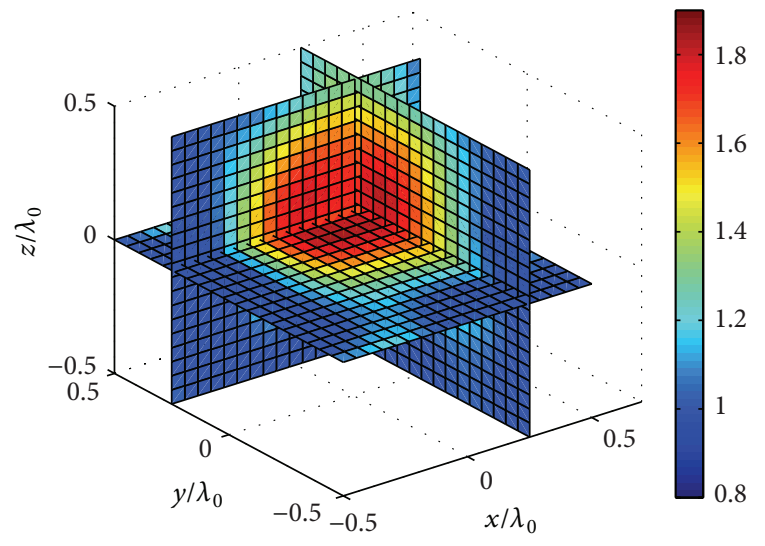

(a)

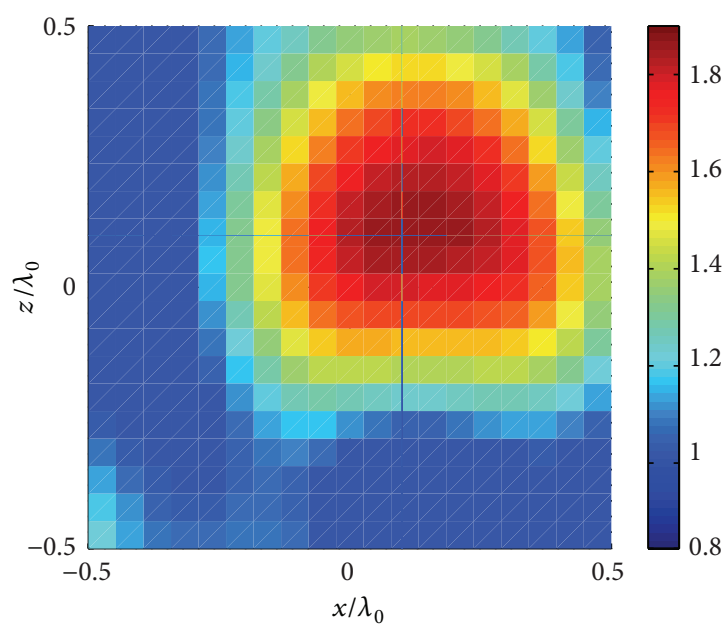

(c)

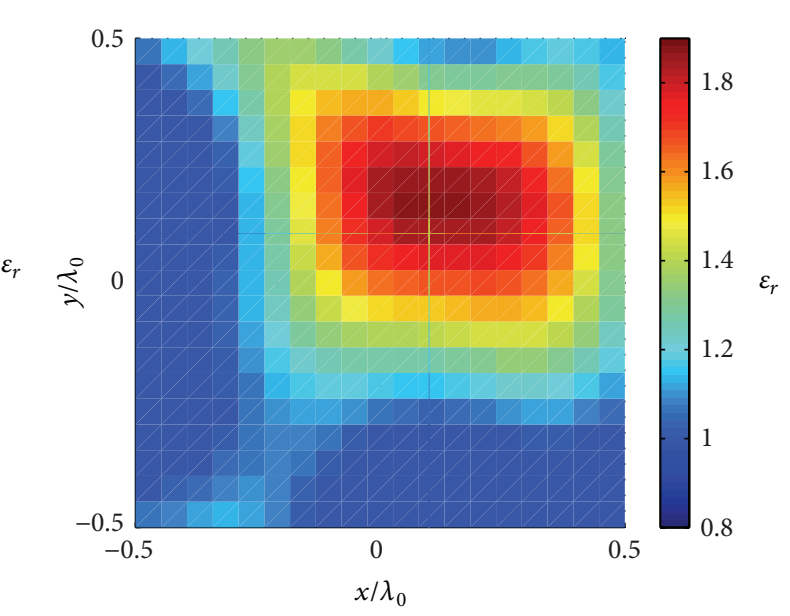

(b)

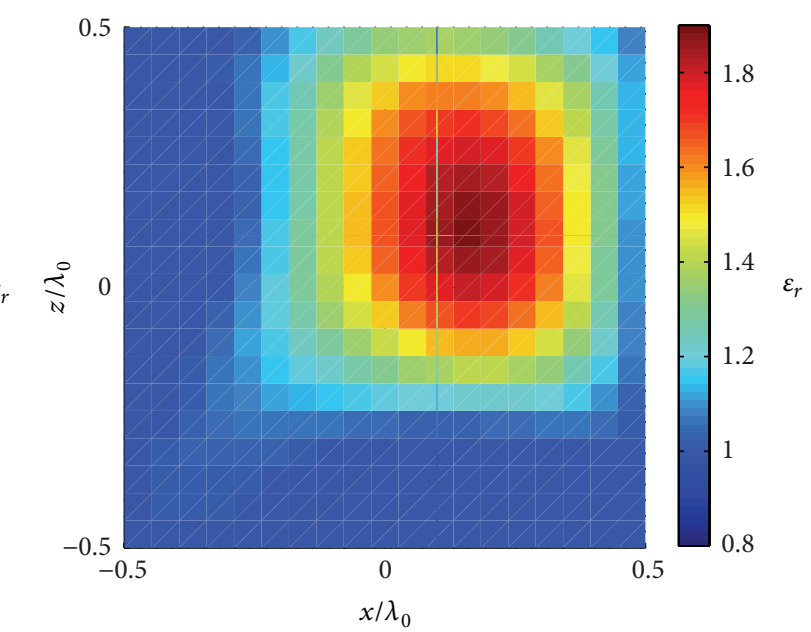

(d)

Figure 3: Reconstructed distribution of the relative dielectric permittivity. (a) 3D view; (b) $x y$ plane $\left(z=0.1 \lambda_{0}\right)$; (c) $x z$ plane $\left(y=0.1 \lambda_{0}\right)$; (d) $y z$ plane $\left(x=0.1 \lambda_{0}\right)$. Homogeneous dielectric cube. $\mathrm{SNR}=20 \mathrm{~dB} . L=3$. 


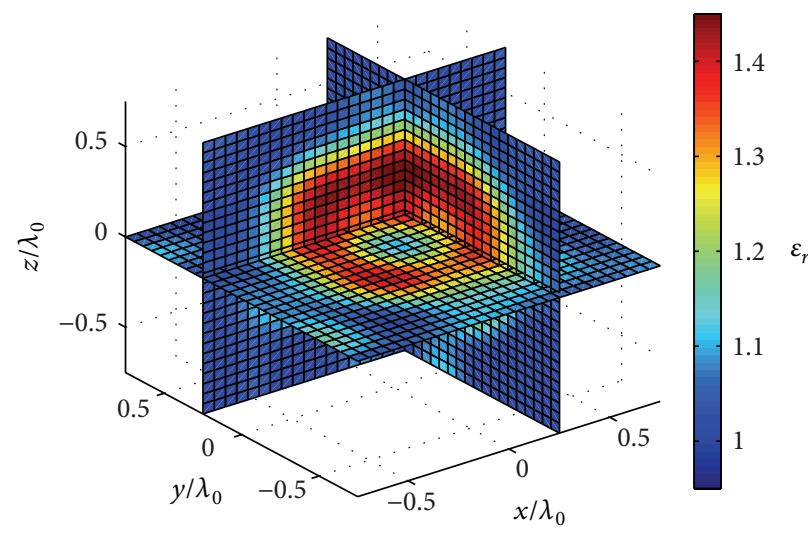

(a)

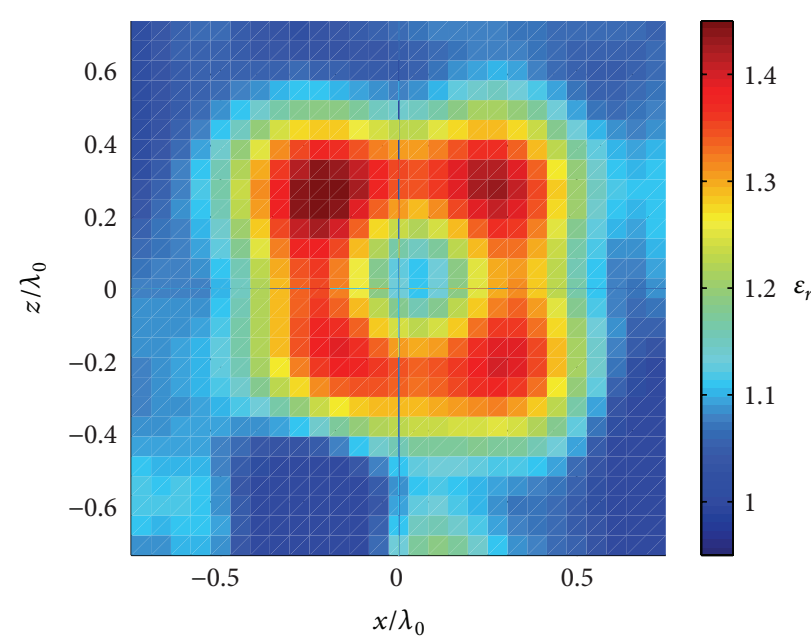

(c)

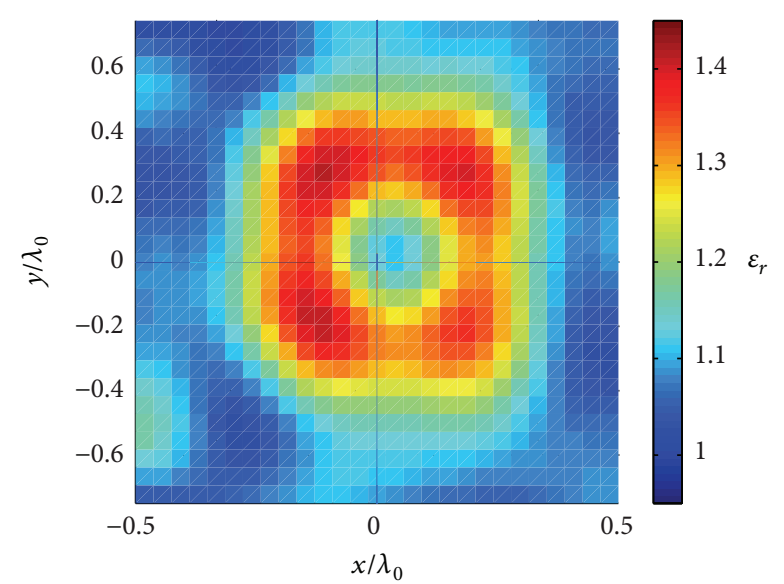

(b)

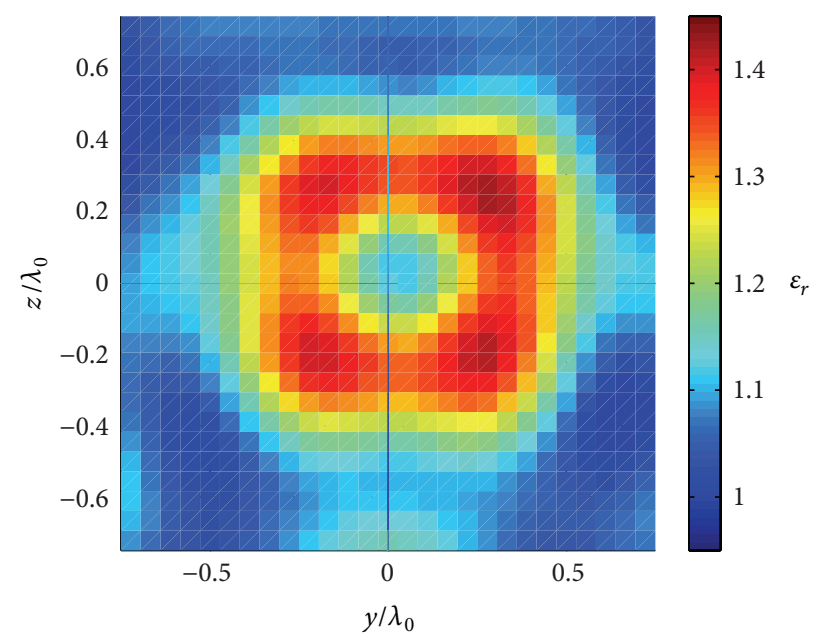

(d)

FIGURE 4: Reconstructed distribution of the relative dielectric permittivity. (a) 3D view; (b) $x y$ plane $(z=0)$; (c) $x z$ plane ( $y=0$ ); (d) $y z$ plane $(x=0)$. Dielectric cube with a void inclusion. $\mathrm{SNR}=25 \mathrm{~dB} . L=2$.

reconstruction of the target, providing a good restoration of the void inclusion too.

In the third case, a more complex configuration with two different separate objects inside the investigation area is assumed. The first one is a cube characterized by $l=$ $0.7 \lambda_{0}$, center $\mathbf{r}_{c}=\left(0.4 \lambda_{0}, 0.4 \lambda_{0}, 0.4 \lambda_{0}\right)$, and $\epsilon_{r}=1.5$. The second one is a sphere with radius $r_{s}=0.4 \lambda_{0}$, centered located at $\mathbf{r}_{s}=\left(0.5 \lambda_{0},-0.5 \lambda_{0},-0.5 \lambda_{0}\right)$, and relative dielectric permittivity $\epsilon_{r}=1.5$. The investigation area is a cubic volume of side $D=2 \lambda_{0}$, which has been partitioned into $N=$ $30 \times 30 \times 30=27000$ subdomains. $N_{S}=6$ views have been used and, for every view, the field is collected in $N_{M}=$ 101 points on a sphere of radius $R_{M}=\sqrt{3} \lambda_{0}$. Similarly to the previous case, the maximum number of allowed outer iterations has been set equal to $K=20$ and the number of inner iterations is $L=2$. The reconstructed distribution of the relative dielectric permittivity is reported in Figure 5. As can be seen from this figure, in this case, too, the two targets are correctly reconstructed. It is worth noting that the two cuts in Figures 5(b) and 5(c) refer to two planes passing only through the first object. Consequently, only the cubic target is visible in these two figures. On the contrary, the $y z$ plane considered in Figure 5(d) contains the both targets.

\section{Conclusions}

In this paper, an algorithm for three-dimensional nondestructive diagnostics through microwave inverse scattering has been analyzed. The dielectric properties of an object (i.e., its contrast function) has to be restored starting from the scattered electric field generated by the interaction with a known electric incident field. The developed algorithm is based on the computation of a regularized solution of the nonlinear equation that relates the scattered electric field in an external observation domain (i.e., the data) with the contrast function in an inaccessible investigation domain (i.e., the unknown). In particular, the approach is based on an outer-inner iterative scheme, where the outer iteration is 


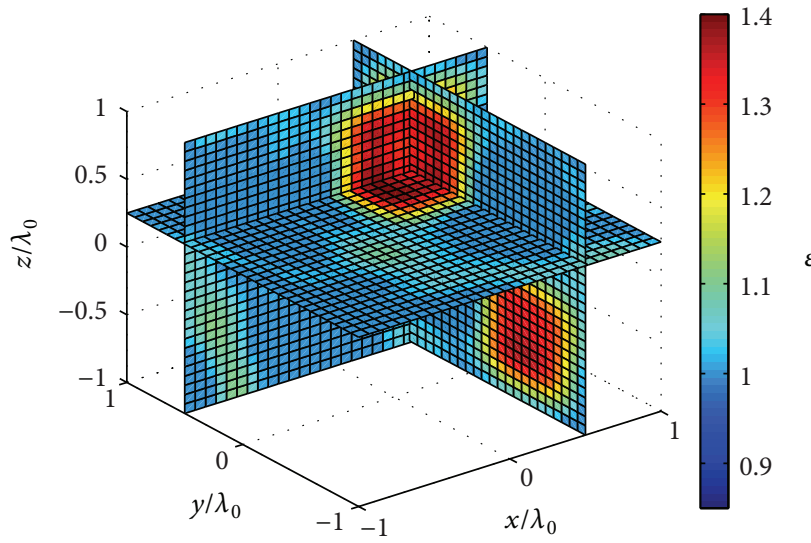

(a)

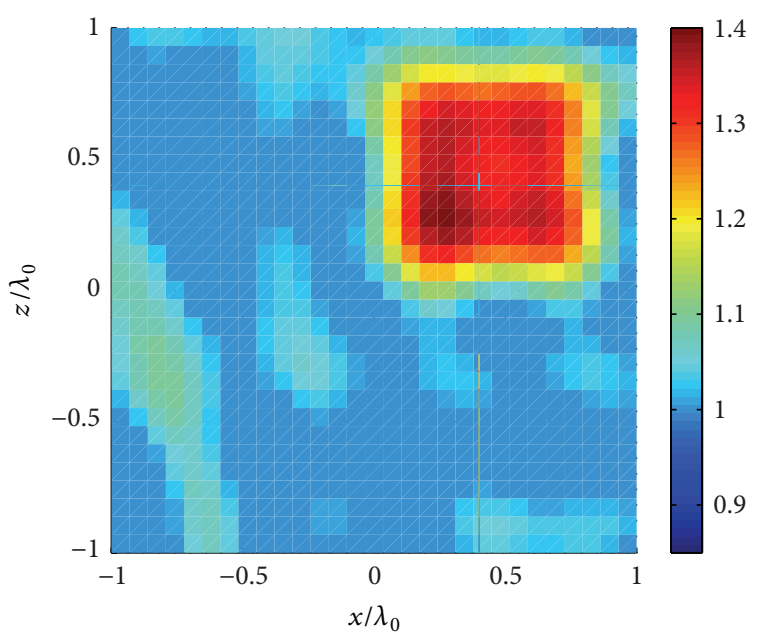

(c)

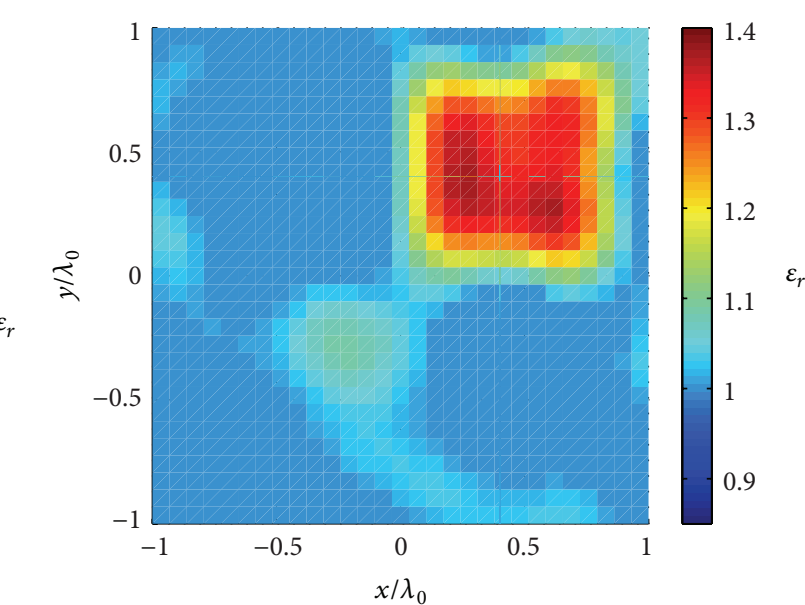

(b)

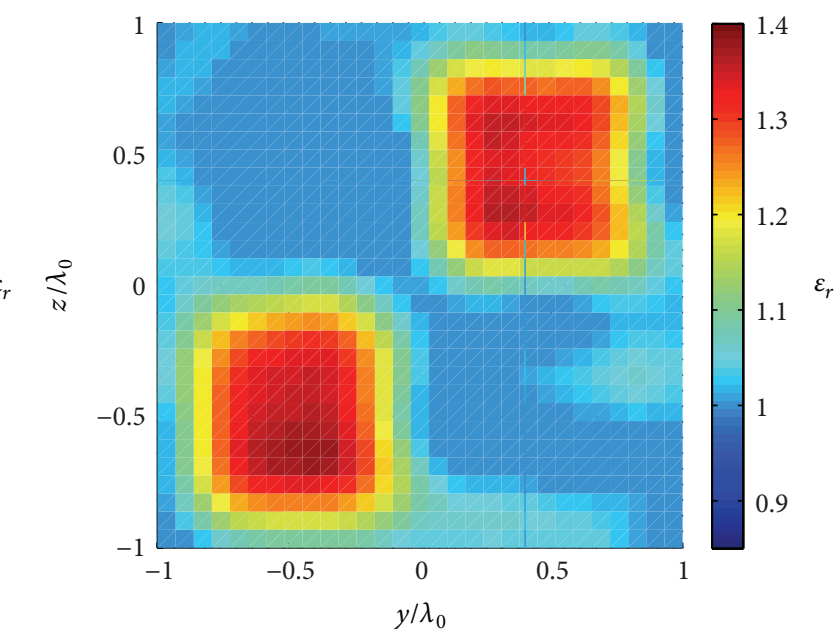

(d)

FiguRE 5: Reconstructed distribution of the relative dielectric permittivity. (a) 3D view; (b) $x y$ plane $\left(z=0.4 \lambda_{0}\right)$; (c) $x z$ plane $\left(y=0.4 \lambda_{0}\right)$; (d) $y z$ plane $\left(x=0.4 \lambda_{0}\right)$. Two separate objects. SNR $=25 \mathrm{~dB} . L=2$.

the basic Newton linearization and the inner iteration is the truncated Landweber method. Several preliminary synthetic numerical tests have been performed in order to validate the developed method. Both homogenous and nonhomogeneous targets have been considered, and in all cases the inversion scheme provided good restorations also for noisy data. A natural subsequent step that will be pursued in the future is the evaluation of the performance of the method against real data.

\section{References}

[1] M. Pastorino, Microwave Imaging, John Wiley, Hoboken, NJ, USA, 2010.

[2] L. L. Monte, D. Erricolo, F. Soldovieri, and M. C. Wicks, "Radio frequency tomography for tunnel detection," IEEE Transactions on Geoscience and Remote Sensing, vol. 48, no. 3, pp. 1128-1137, 2010.

[3] R. Persico and F. Soldovieri, "Two-dimensional linear inverse scattering for dielectric and magnetic anomalies," Near Surface Geophysics, vol. 9, no. 3, pp. 287-295, 2011.
[4] C. Yu, M. Yuan, and Q. H. Liu, "Reconstruction of 3D objects from multi-frequency experimental data with a fast DBIMBCGS method," Inverse Problems, vol. 25, no. 2, Article ID 024007, 2009.

[5] F. Li, Q. H. Liu, and L.-P. Song, "Three-dimensional reconstruction of objects buried in layered media using Born and distorted Born iterative methods," IEEE Geoscience and Remote Sensing Letters, vol. 1, no. 2, pp. 107-111, 2004.

[6] R. Solimene, A. Brancaccio, R. Di Napoli, and R. Pierri, "3D sliced tomographic inverse scattering experimental results," Progress in Electromagnetics Research, vol. 105, pp. 1-13, 2010.

[7] R. Solimene, R. Di Napoli, F. Soldovieri, and R. Pierri, “TWI for an unknown symmetric lossless wall," IEEE Transactions on Geoscience and Remote Sensing, vol. 49, no. 8, pp. 2876-2886, 2011.

[8] A. Randazzo, "Swarm optimization methods in microwave imaging," International Journal of Microwave Science and Technology, vol. 2012, Article ID 491713, 12 pages, 2012.

[9] G. Bozza and M. Pastorino, "An inexact Newton-based approach to microwave imaging within the contrast source 
formulation," IEEE Transactions on Antennas and Propagation, vol. 57, no. 4, pp. 1122-1132, 2009.

[10] D. W. Winters, B. D. Van Veen, and S. C. Hagness, "A sparsity regularization approach to the electromagnetic inverse scattering problem," IEEE Transactions on Antennas and Propagation, vol. 58, no. 1, pp. 145-154, 2010.

[11] M. Donelli, A. Massa, G. Oliveri, M. Pastorino, and A. Randazzo, "A differential evolution-based iterative multi-scaling algorithm for microwave imaging of dielectric structures," in Proceedings of the IEEE International Conference on Imaging Systems and Techniques (IST '10), pp. 90-95, July 2010.

[12] R. D. Monleone, M. Pastorino, J. Fortuny-Guasch et al., "Impact of background noise on dielectric reconstructions obtained by a prototype of microwave axial tomograph," IEEE Transactions on Instrumentation and Measurement, vol. 61, no. 1, pp. 140-148, 2012.

[13] A. Franchois and C. Pichot, "Microwave imaging-complex permittivity reconstruction with a levenberg-marquardt method," IEEE Transactions on Antennas and Propagation, vol. 45, no. 2, pp. 203-215, 1997.

[14] A. Randazzo, G. Oliveri, A. Massa, and M. Pastorino, "Electromagnetic inversion with the multiscaling inexact Newton method-experimental validation," Microwave and Optical Technology Letters, vol. 53, no. 12, pp. 2834-2838, 2011.

[15] M. J. Akhtar and A. S. Omar, "Reconstructing permittivity profiles using integral transforms and improved renormalization techniques," IEEE Transactions on Microwave Theory and Techniques, vol. 48, no. 8, pp. 1385-1393, 2000.

[16] M. Brignone, G. Bozza, R. Aramini, M. Pastorino, and M. Piana, "A fully no-sampling formulation of the linear sampling method for three-dimensional inverse electromagnetic scattering problems," Inverse Problems, vol. 25, no. 1, Article ID 015014, 2009.

[17] G. Bozza, C. Estatico, M. Pastorino, and A. Randazzo, "Microwave imaging for nondestructive testing of dielectric structures: Numerical simulations using an inexact Newton technique," Materials Evaluation, vol. 65, no. 9, pp. 917-922, 2007.

[18] G. Bozza, M. Brignone, and M. Pastorino, "Application of the no-sampling linear sampling method to breast cancer detection," IEEE Transactions on Biomedical Engineering, vol. 57, no. 10, pp. 2525-2534, 2010.

[19] D. K. Ghodgaonkar, O. P. Gandhi, and M. J. Hagmann, "Estimation of Complex permittivities of three-dimensional inhomogeneous biological bodies," IEEE Transactions on Microwave Theory and Techniques, vol. 31, no. 6, pp. 442-446, 1983.

[20] S. Caorsi, G. L. Gragnani, and M. Pastorino, "Equivalent current density reconstruction for microwave imaging purposes," IEEE Transactions on Microwave Theory and Techniques, vol. 37, no. 5, pp. 910-916, 1989.

[21] G. G. Cook, A. P. Anderson, A. J. T. Whitaker, and J. C. Bennett, "High resolution three-dimensional microwave imaging of antennas," IEEE Transactions on Antennas and Propagation, vol. 37, no. 6, pp. 768-779, 1989.

[22] W. W. Guo and T. C. Guo, "Three-dimensional dielectric imaging by microwave inverse scattering with resolution unlimited by wavelength," in Proceedings of the IEEE Annual Report: Conference on Electrical Insulation and Dielectric Phenomena, pp. 65-74, Leesburg, Va, USA, November 1989.

[23] M. F. Adams and A. P. Anderson, "Three-dimensional image-construction technique and its application to coherent microwave diagnostics," IEE Proceedings $H$, vol. 127, no. 3, pp. 138-142, 1980.
[24] S. Y. Semenov, R. H. Svenson, A. E. Souvorov et al., "Microwave tomography. Experimental imaging on two- and threedimensional systems," in Proceedings of the IEEE MTT-S International Microwave Symposium, vol. 2, pp. 763-766, June 1998.

[25] J.-H. Lin and W. C. Chew, "Three-dimensional microwave imaging by local shape function method with CGFFT," in Proceedings of the AP-S International Symposium \& URSI Radio Science Meeting, pp. 2148-2151, Baltimore, Md, USA, July 1996.

[26] S. Caorsi, G. L. Gragnani, and M. Pastorino, "Redundant electromagnetic data for microwave imaging of three-dimensional dielectric objects," IEEE Transactions on Antennas and Propagation, vol. 42, no. 5, pp. 581-589, 1994.

[27] J.-C. Bolomey and L. Jofre, "Three decades of active microwave imaging achievements, difficulties and future challenges," in Proceedings of the IEEE International Conference on Wireless Information Technology and Systems (ICWITS '10), Honolulu, Hawaii, USA, September 2010.

[28] A. Joisel, J.-M. Geffrin, J. M. Elissalt, K. Belkebir, N. Joachimowicz, and J. C. Bolomey, "Iterative $2 \mathrm{D}$ reconstruction algorithms for microwave active imaging systems," in Proceedings of the IEE Electronics Division Colloquium on The Applications of Microwaves in Medicine, pp. 9/1-9/4, London, UK, February 1995.

[29] T. Belkebir, C. Pichot, J. C. Bolomey et al., "Microwave tomography system for reinforced concrete structures," in Proceedings of the 24th European Microwave Conference, vol. 2, pp. 1209-1214, Cannes, France, 1994.

[30] Á. Díaz-Bolado, T. Henriksson, P.-A. Barrière et al., "Towards a planar microwave tomography system for early stage breast cancer detection," in Proceedings of the 30th URSI General Assembly and Scientific Symposium (URSIGASS '11), Istanbul, Turkey, August 2011.

[31] M. Benedetti, M. Donelli, A. Martini, M. Pastorino, A. Rosani, and A. Massa, "An innovative microwave-imaging technique for nondestructive evaluation: applications to civil structures monitoring and biological bodies inspection," IEEE Transactions on Instrumentation and Measurement, vol. 55, no. 6, pp. 1878-1883, 2006.

[32] G. Oliveri, L. Lizzi, M. Pastorino, and A. Massa, "A nested multi-scaling inexact-newton iterative approach for microwave imaging," IEEE Transactions on Antennas and Propagation, vol. 60, no. 2, pp. 971-983, 2012.

[33] T. Henriksson, N. Joachimowicz, C. Conessa, and J.-C. Bolomey, "Quantitative microwave imaging for breast cancer detection using a planar $2.45 \mathrm{GHz}$ system," IEEE Transactions on Instrumentation and Measurement, vol. 59, no. 10, pp. 26912699, 2010.

[34] M. Pastorino, S. Caorsi, A. Massa, and A. Randazzo, "Reconstruction algorithms for electromagnetic imaging," IEEE Transactions on Instrumentation and Measurement, vol. 53, no. 3, pp. 692-699, 2004.

[35] J.-H. Lin, C.-C. Lu, Y. M. Wang, and W. C. Chew, "Processing microwave experimental data with the distorted born iterative method of nonlinear inverse scattering," in Proceedings of the IEEE Antennas and Propagation International Symposium, pp. 500-503, Ann Arbor, Mich, USA, July 1993.

[36] J. M. Rius, C. Pichot, L. Jofre et al., "Planar and cylindrical active microwave temperature imaging: numerical simulations," IEEE Transactions on Medical Imaging, vol. 11, no. 4, pp. 457-469, 1992.

[37] R. Solimene, G. Ruvio, R. Pierri, and M. J. Ammann, "Microwave focusing algorithms for breast cancer detection: a 
comparison for a 2D simplified scenario," in Proceedings of the 5th European Conference on Antennas and Propagation (EUCAP '11), p. 289, Rome, Italy, April 2011.

[38] R. Solimene, F. Soldovieri, A. Baratonia, and R. Pierri, "Experimental validation of a linear inverse scattering TWI algorithm by a SF-CW radar," IEEE Antennas and Wireless Propagation Letters, vol. 9, pp. 506-509, 2010.

[39] S. Caorsi, A. Massa, M. Pastorino, M. Raffetto, and A. Randazzo, "Detection of buried inhomogeneous elliptic cylinders by a memetic algorithm," IEEE Transactions on Antennas and Propagation, vol. 51, no. 10, pp. 2878-2884, 2003.

[40] A. Brancaccio, G. Leone, F. Soldovieri, and R. Pierri, "Localization of interfaces embedded in a half-space by a linear inverse scattering algorithm," IEEE Transactions on Geoscience and Remote Sensing, vol. 45, no. 11, pp. 3661-3671, 2007.

[41] R. Pierri, F. De Blasio, and A. Brancaccio, "Multifrequency approach to inverse scattering: the linear and quadratic models," in Proceedings of the IEEE International Geoscience and Remote Sensing Symposium (IGARSS '99), pp. 2522-2524, Hamburg, Germany, July 1999.

[42] R. Pierri, G. Leone, and R. Persico, "Quadratic models inverse in scattering: numerical experiments," in Proceedings of the 1999 IEEE International Geoscience and Remote Sensing Symposium (IGARSS '99), pp. 1404-1406, Hamburg, Germany, July 1999.

[43] A. Salvade, M. Pastorino, R. Monleone, G. Bozza, and A. Randazzo, "A new microwave axial tomograph for the inspection of dielectric materials," IEEE Transactions on Instrumentation and Measurement, vol. 58, no. 7, pp. 2072-2079, 2009.

[44] Y. Liu, L. Zhu, and D. Huang, "A two-dimensional microwave radiation imaging method based on data fusion," in Proceedings of the 10th International Symposium on Antennas, Propagation, and EM Theory (ISAPE '12), pp. 600-603, Xian, China, 2012.

[45] L. Kun, D. Guohong, and L. Cheng, "Monostatic microwave imaging of $2 \mathrm{D}$ conductor based on Time-Domain Finite Element," in Proceedings of the 2010 International Conference on Microwave and Millimeter Wave Technology (ICMMT '10), pp. 820-822, Chengdu, China, May 2010.

[46] G. Oliveri, A. Randazzo, M. Pastorino, and A. Massa, "Imaging of separate scatterers by means of a multiscaling multiregion Inexact-Newton approach," Progress In Electromagnetics Research M, vol. 18, pp. 247-257, 2011.

[47] T. Wei, C. Liao, and Y. Liu, "Application of Micro-Genetic Algorithm (MGA) to monostatic microwave local imaging of PEC cylinder," in Proceedings of the IEEE Antennas and Propagation Society International Symposium (APS '06), pp. 1081-1083, Albuquerque, NM, USA, July 2006.

[48] G. Oliveri, A. Randazzo, M. Pastorino, and A. Massa, "Electromagnetic imaging within the contrast-source formulation by means of the multiscaling inexact Newton method," The Journal of the Optical Society of America, vol. 29, no. 6, p. 945, 2012.

[49] S. Caorsi, A. Costa, and M. Pastorino, "Microwave imaging within the second-order born approximation: stochastic optimization by a genetic algorithm," IEEE Transactions on Antennas and Propagation, vol. 49, no. 1, pp. 22-31, 2001.

[50] C. Yu, M. Yuan, J. Stang et al., "Active microwave imaging II: 3-D system prototype and image reconstruction from experimental data," IEEE Transactions on Microwave Theory and Techniques, vol. 56, no. 4, pp. 991-1000, 2008.

[51] R. K. Amineh, A. Khalatpour, and N. K. Nikolova, "Threedimensional microwave holographic imaging using co- and cross-polarized data," IEEE Transactions on Antennas and Propagation, vol. 60, no. 7, pp. 3526-3531, 2012.
[52] A. H. Golnabi, P. M. Meaney, N. R. Epstein, and K. D. Paulsen, "Microwave imaging for breast cancer detection: advances in three Dimensional image reconstruction," in Proceedings of the 33rd Annual International Conference of the IEEE Engineering in Medicine and Biology Society (EMBS '11), pp. 5730-5731, Boston, Mass, USA, September 2011.

[53] A. Sabouni, M. Ostadrahimi, S. Noghanian, and M. Pavlovic, "Three-dimensional accurate modeling of the microwave tomography imaging system," in Proceedings of the IEEE International Symposium on Antennas and Propagation and USNC/URSI National Radio Science Meeting, (APSURSI '11), pp. 2557-2560, Spokane, Wash, USA, July 2011.

[54] V.Zhurbenko, T. Rubæk, V. Krozer, and P. Meincke, "Design and realisation of a microwave three-dimensional imaging system with application to breast-cancer detection," IET Microwaves, Antennas and Propagation, vol. 4, no. 12, pp. 2200-2211, 2010.

[55] A. Abubakar and T. M. Habashy, "Multiplicative regularized Gauss-Newton approach for three-dimensional microwave imaging," in Proceedings of the IEEE MTT-S International Microwave Symposium (MTT '10), pp. 1596-1599, Anaheim, Calif, USA, May 2010.

[56] P. C. Chaumet, K. Belkebir, and A. Sentenac, "Experimental microwave imaging of three-dimensional targets with different inversion procedures," Journal of Applied Physics, vol. 106, no. 3, Article ID 034901, 2009.

[57] M. Donelli, D. Franceschini, P. Rocca, and A. Massa, "Threedimensional microwave imaging problems solved through an efficient multiscaling particle swarm optimization," IEEE Transactions on Geoscience and Remote Sensing, vol. 47, no. 5, pp. 1467-1481, 2009.

[58] D. W. Winters, J. D. Shea, P. Kosmas, B. D. Van Veen, and S. C. Hagness, "Three-dimensional microwave breast imaging: dispersive dielectric properties estimation using patient-specific basis functions," IEEE Transactions on Medical Imaging, vol. 28, no. 7, pp. 969-981, 2009.

[59] P. Kosmas, J. D. Shea, B. D. Van Veen, and S. C. Hagness, “Threedimensional microwave imaging of realistic breast phantoms via an inexact Gauss-Newton algorithm," in Proceedings of the 2008 IEEE International Symposium on Antennas and Propagation and USNC/URSI National Radio Science Meeting (APSURSI '08), San Diego, Calif, USA, July 2008.

[60] G. Liu and Y. Zhang, "Three-dimensional microwave imaging for breast cancer detection based on forward-backward timestepping and Tikhonov's regularization methods," in Proceedings of the IEEE 12th International Conference on Communication Technology (ICCT '10), pp. 845-848, Nanjing, China, November 2010.

[61] T. Rubaek, P. Meincke, O. S. Kim, P. M. Meaney, and K. D. Paulsen, "Application of the log-phase formulation for threedimensional microwave imaging," in Proceedings of the 2nd European Conference on Antennas and Propagation (EuCAP '07), Edinburgh, UK, November 2007.

[62] J. De Zaeytijd, A. Franchois, C. Eyraud, and J.-M. Geffrin, "Fullwave three-dimensional microwave imaging with a regularized Gauss-Newton method-theory and experiment," IEEE Transactions on Antennas and Propagation, vol. 55, no. 11, pp. 32793292, 2007.

[63] P. Rashidi, M. El-Shenawee, D. Macías, and E. Miller, "Microwave imaging of three-dimensional dielectric objects employing evolution strategies," in Proceedings of the IEEE/ACES International Conference on Wireless Communications and Applied Computational Electromagnetics, pp. 393-396, Honolulu, Hawaii, USA, April 2005. 
[64] H. Chen and J. Miao, "Sampling in three-dimension microwave near-field imaging," in Proceedings of the Asia-Pacific Microwave Conference (APMC '05), Suzhou, China, December 2005.

[65] Z. Q. Zhang and Q. H. Liu, "Three-dimensional nonlinear image reconstruction for microwave biomedical imaging," IEEE Transactions on Biomedical Engineering, vol. 51, no. 3, pp. 544548, 2004.

[66] M. Brignone, G. Bozza, A. Randazzo, M. Piana, and M. Pastorino, "A hybrid approach to 3D microwave imaging by using linear sampling and ACO," IEEE Transactions on Antennas and Propagation, vol. 56, no. 10, pp. 3224-3232, 2008.

[67] S. Y. Semenov, A. E. Bulyshev, A. E. Souvorov et al., "Threedimensional microwave tomography: experimental imaging of phantoms and biological objects," IEEE Transactions on Microwave Theory and Techniques, vol. 48, no. 6, pp. 1071-1074, 2000.

[68] Q.-H. Liu, C. Yu, J. Stang et al., "Experimental and numerical investigations of a high-resolution 3D microwave imaging system for breast cancer detection," in Proceedings of the IEEE Antennas and Propagation Society International Symposium (AP-S '07), p. 2192, Honolulu, Hawaii, USA, June 2007.

[69] A. Abubakar, T. M. Habashy, G. Pan, and M. K. Li, "Application of the multiplicative regularized gauss-newton algorithm for three-dimensional microwave imaging," IEEE Transactions on Antennas and Propagation, vol. 60, no. 5, pp. 2431-2441, 2012.

[70] C. Estatico, G. Bozza, A. Massa, M. Pastorino, and A. Randazzo, "A two-step iterative inexact-Newton method for electromagnetic imaging of dielectric structures from real data," Inverse Problems, vol. 21, no. 6, pp. S81-S94, 2005.

[71] C. Estatico, M. Pastorino, and A. Randazzo, "An inexactNewton method for short-range microwave imaging within the second-order born approximation," IEEE Transactions on Geoscience and Remote Sensing, vol. 43, no. 11, pp. 2593-2605, 2005.

[72] G. Bozza, C. Estatico, M. Pastorino, and A. Randazzo, "An inexact Newton method for microwave reconstruction of strong scatterers," IEEE Antennas and Wireless Propagation Letters, vol. 5, no. 1, pp. 61-64, 2006.

[73] C. Estatico, M. Pastorino, and A. Randazzo, "A novel microwave imaging approach based on regularization in Lp Banach spaces," IEEE Transactions on Antennas and Propagation, vol. 60, no. 7, pp. 3373-3381, 2012.

[74] G. Bozza, C. Estatico, M. Pastorino, and A. Randazzo, "Application of an inexact-Newton method within the second-order Born approximation to buried objects," IEEE Geoscience and Remote Sensing Letters, vol. 4, no. 1, pp. 51-55, 2007.

[75] R. Harrington, Field Computation by Moment Methods, IEEE Press, Piscataway, NJ, USA, 1993.

[76] D. E. Livesay and K. M. Chen, "Electromagnetic field induced inside arbitrarily shaped biological bodies," IEEE Transactions on Microwave Theory and Techniques, vol. 22, no. 12, pp. 12731280, 1974.

[77] X. M. Xu, Q. H. Liu, and Z. Q. Zhang, "The stabilized biconjugate gradient fast Fourier transform method for electromagnetic scattering," Applied Computational Electromagnetics Society Newsletter, vol. 17, no. 1, pp. 97-103, 2002.

[78] X. Xu, Q. H. Liu, and Z. Q. Zhang, “The stabilized biconjugate gradient fast Fourier transform method for electromagnetic scattering," in Proceedings of the IEEE Antennas and Propagation Society International Symposium, pp. 614-617, San Antonio, Tex, USA, June 2002.
[79] M. Bertero and P. Boccacci, Introduction to Inverse Problems in Imaging, IOP, Bristol, UK, 1998. 

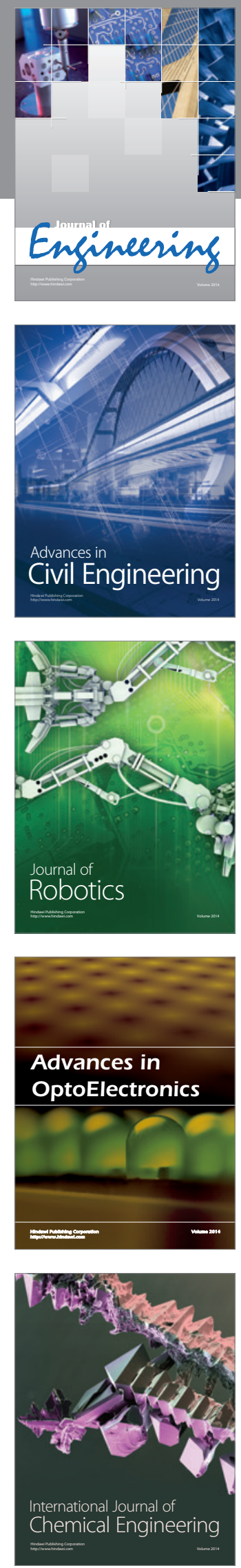

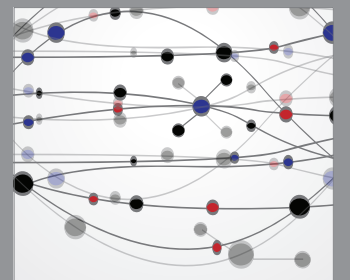

The Scientific World Journal
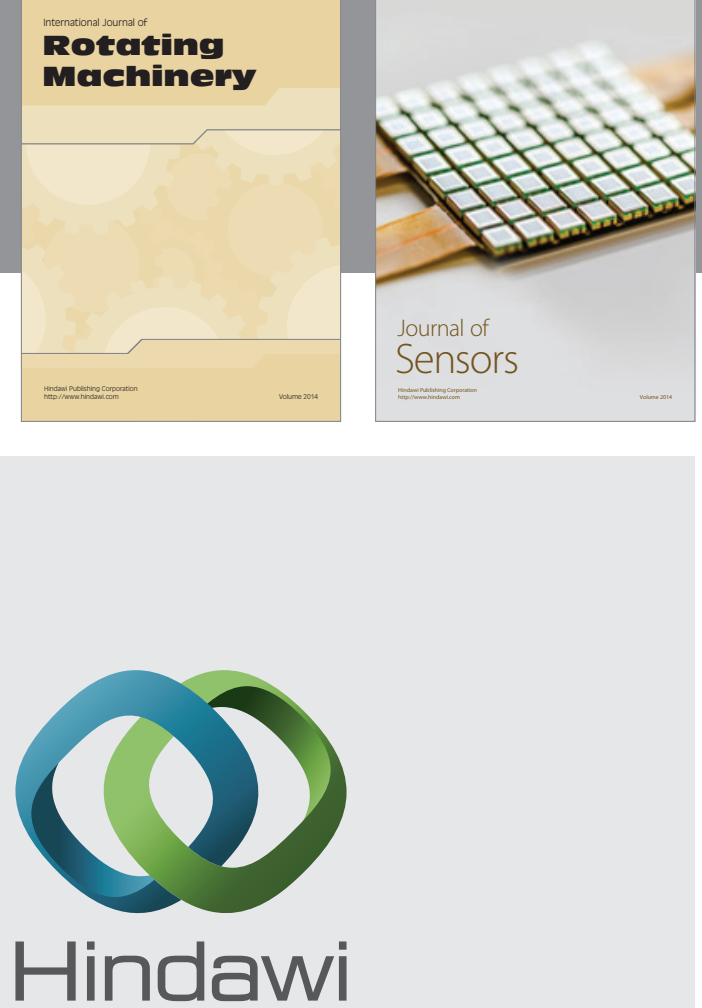

Submit your manuscripts at http://www.hindawi.com
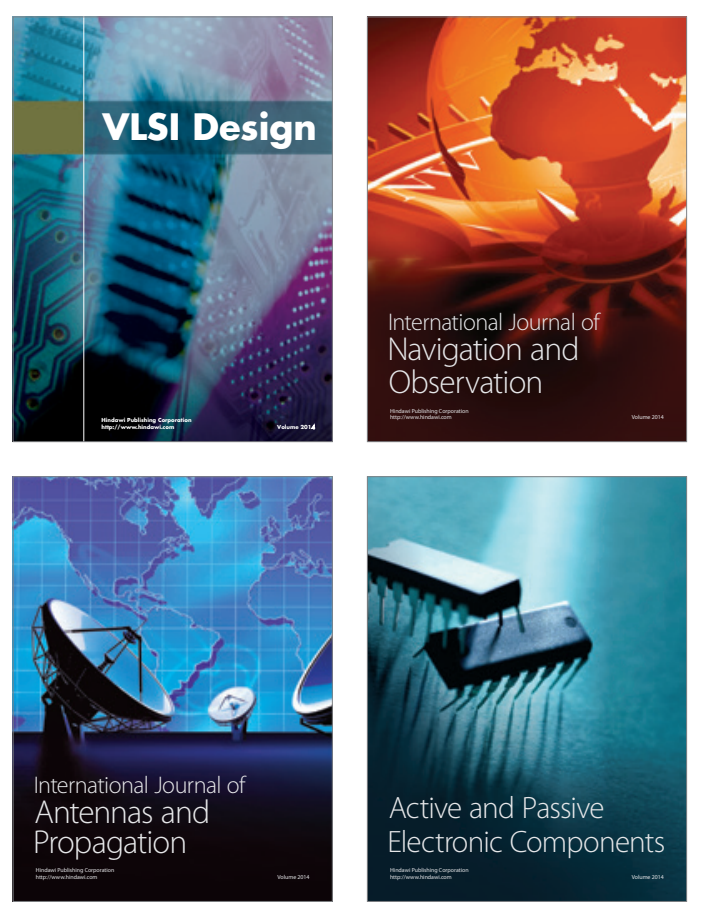
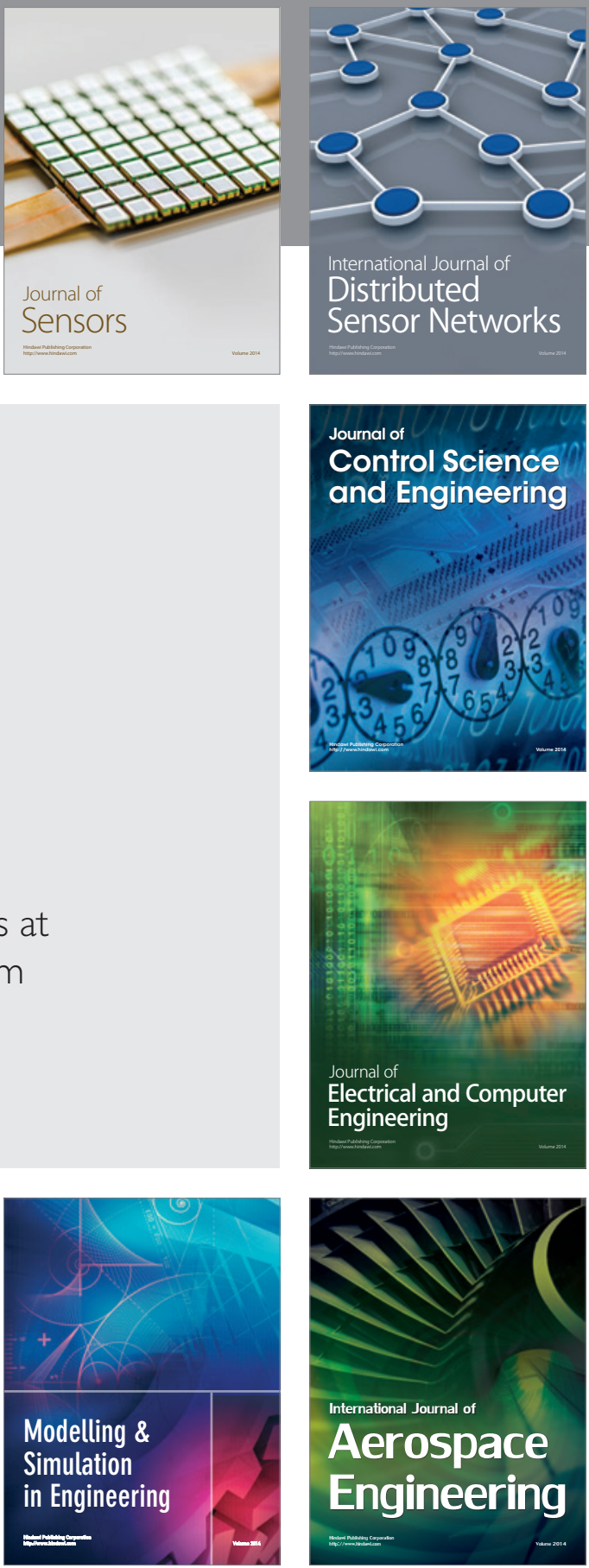

Journal of

Control Science

and Engineering
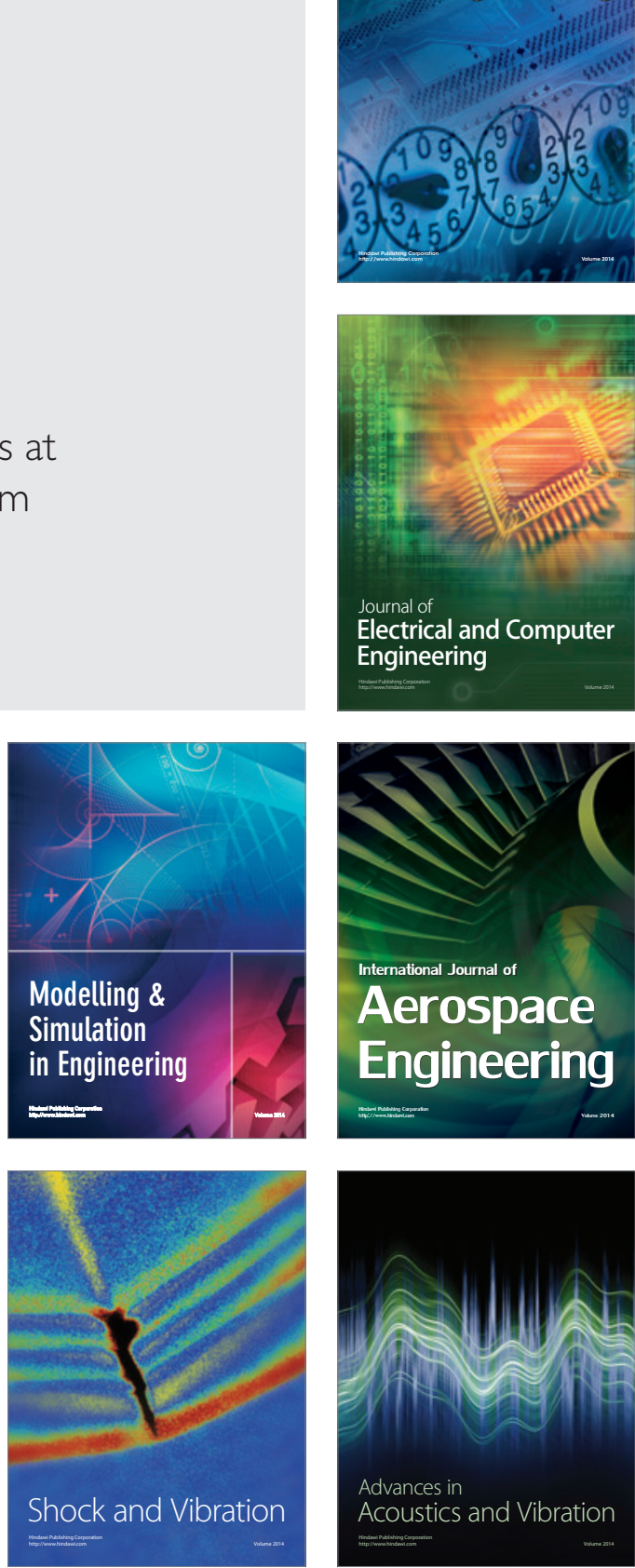